\title{
CORRECTION
}

View Article Online

View Journal I View Issue

Check for updates

Cite this: J. Mater. Chem. C, 2019,

7, 14395

DOI: $10.1039 / c 9 t c 90229 e$

\section{Correction: Electrochromic triphenylamine-based cobalt(II) complex nanosheets}

\author{
Yurong Liu, ${ }^{a b c}$ Ryota Sakamoto, ${ }^{d}$ Cheuk-Lam Ho, ${ }^{\text {ab }}$ Hiroshi Nishihara*d and \\ Wai-Yeung Wong*abc \\ Correction for 'Electrochromic triphenylamine-based cobalt(॥) complex nanosheets' by Yurong Liu et al., \\ J. Mater. Chem. C, 2019, 7, 9159-9166.
}

rsc.li/materials-c

The authors would like to correct the affiliation of the authors Wai-Yeung Wong and Yurong Liu as follows:

${ }^{\mathrm{c}}$ Institute of Molecular Functional Materials, Department of Chemistry, Hong Kong Baptist University, Waterloo Road, Kowloon Tong, Hong Kong and Hong Kong Baptist University Institute of Research and Continuing Education, Shenzhen Virtual University Park, Shenzhen 518057, P. R. China.

The correct author and affiliations list is as above.

The Acknowledgements originally published for this article were incomplete. The following information should be added: W.-Y.W. also thanks the financial support from the National Natural Science Foundation of China (51573151).

The Royal Society of Chemistry apologises for these errors and any consequent inconvenience to authors and readers.

\footnotetext{
${ }^{a}$ The Hong Kong Polytechnic University Shenzhen Research Institute, Shenzhen 518057, P. R. China. E-mail: wai-yeung.wong@polyu.edu.hk

${ }^{b}$ Department of Applied Biology and Chemical Technology, The Hong Kong Polytechnic University, Hong Hom, Hong Kong, P. R. China. E-mail: cheuk-lam.ho@polyu.edu.hk ${ }^{c}$ Institute of Molecular Functional Materials, Department of Chemistry, Hong Kong Baptist University, Waterloo Road, Kowloon Tong, Hong Kong and Hong Kong Baptist University Institute of Research and Continuing Education, Shenzhen Virtual University Park, Shenzhen 518057, P. R. China

${ }^{d}$ Department of Chemistry, Graduate School of Science, The University of Tokyo, 7-3-1, Hongo, Bunkyo-ku, Tokyo 113-0033, Japan. E-mail: nisihara@chem.s.u-tokyo.ac.jp
} 